\title{
Qualitative adaptation of child behaviour problem instruments in a developing-country setting
}

\author{
B. Khan ${ }^{7}$ and B.I. Avan ${ }^{2}$
}

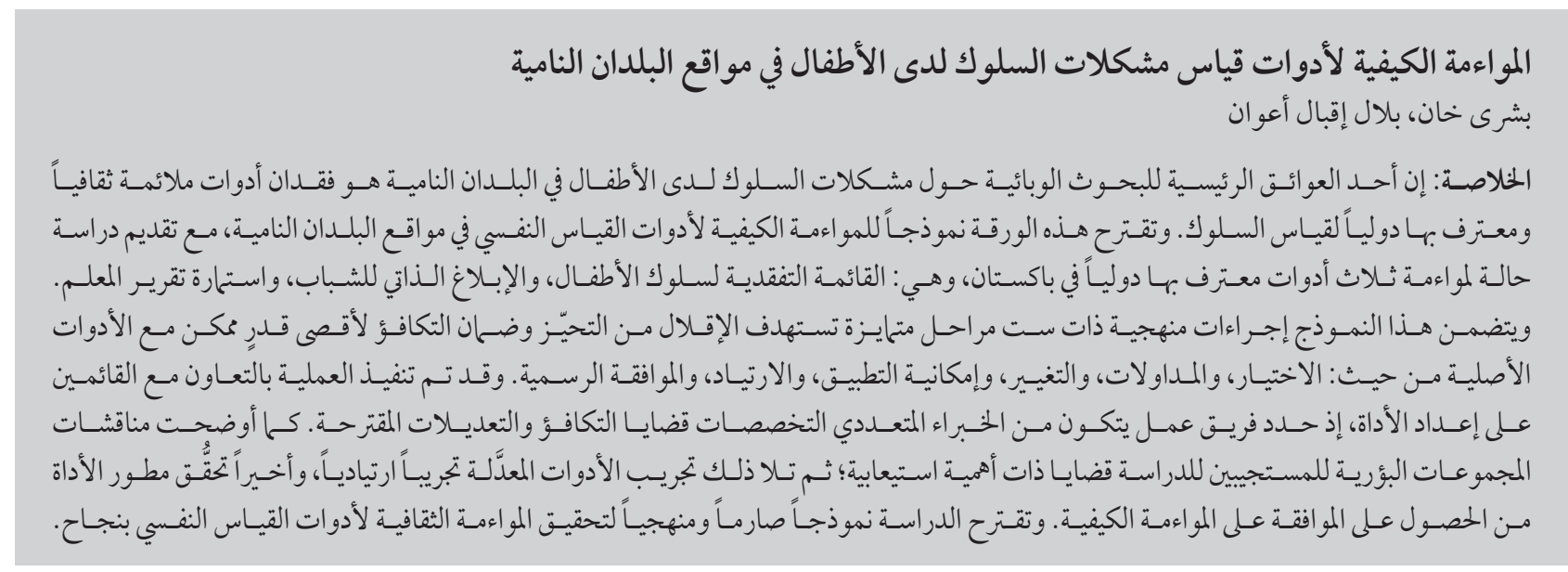

ABSTRACT A key barrier to epidemiological research on child behaviour problems in developing countries is the lack of culturally relevant, internationally recognized psychometric instruments. This paper proposes a model for the qualitative adaptation of psychometric instruments in developing-country settings and presents a case study of the adaptation of 3 internationally recognized instruments in Pakistan: the Child Behavior Checklist, the Youth Self-Report and the Teacher's Report Form. This model encompassed a systematic procedure with 6 distinct phases to minimize bias and ensure equivalence with the original instruments: selection, deliberation, alteration, feasibility, testing and formal approval. The process was conducted in collaboration with the instruments' developer. A multidisciplinary working group of experts identified equivalence issues and suggested modifications. Focus group discussions with informants highlighted comprehension issues. Subsequently modified instruments were thoroughly tested. Finally, the instruments' developer approval further validated the qualitative adaptation. The study proposes a rigorous and systematic model to effectively achieve cultural adaptation of psychometric instruments.

Adaptation qualitative des instruments mesurant les problèmes comportementaux chez l'enfant dans un contexte de pays en développement

RÉSUMÉ Le manque d'instruments psychométriques internationalement reconnus et culturellement adaptés représente un obstacle majeur à la recherche épidémiologique sur les problèmes comportementaux chez l'enfant dans les pays en développement. Le présent article propose un modèle pour l'adaptation qualitative d'instruments psychométriques au contexte des pays en développement et présente une étude de cas sur l'adaptation au Pakistan de trois instruments internationalement reconnus : la liste de contrôle du comportement des enfants (Child Behavior Checklist), l'auto-évaluation des jeunes (Youth Self-Report) et le rapport d'évaluation de l'enseignant (Teacher's Report Form). Le modèle comprenait une méthode systhématique en six phases distinctes visant à réduire au minimum les biais et à garantir l'équivalence avec les instruments originaux : sélection, délibération, modification, faisabilité, test et approbation formelle. La méthode a été utilisée en collaboration avec le concepteur des instruments. Un groupe de travail pluridisciplinaire composé d'experts a identifié les problèmes d'équivalence puis a suggéré des modifications. Des discussions entre les groupes thématiques et les informateurs ont permis de mettre en évidence les problèmes de compréhension. Les instruments modifiés ont ultérieurement été entièrement testés. Enfin, l'approbation du concepteur des instruments a aussi permis de valider l'adaptation qualitative. L'étude propose un modèle rigoureux et complet permettant d'obtenir une adaptation culturelle efficace des instruments psychométriques.

'Department of Psychology, University of Karachi, Karachi, Pakistan (Correspondence to B. Khan: bushra.khan@ymail.com).

${ }^{2}$ London School of Hygiene and Tropical Medicine, London, United Kingdom.

Received: 25/08/13; accepted: 23/03/14 


\section{Introduction}

Behaviour and emotional problems in children are becoming a globally importantpublichealthissue (1-3). Behaviour problems have long-term detrimental consequences (4-7); early detection of problems, followed by evidence-based interventions, is vital for their prevention (1). Epidemiological studies show that the prevalence of childhood behaviour problems varies cross-culturally, although most of this evidence comes from developed countries (8-14). The key barrier to collecting empirical evidence in developing countries is the unavailability of culturally and scientifically adapted instruments (15) to measure the burden of problems and to track the effectiveness of interventions. The aim of adaptation is to make an instrument relevant to a culture that is different from the one for which it was originally developed. It limits any partiality and unfairness of the tool with a particular focus on construct bias, i.e. when concepts differ cross-culturally; on method bias, i.e. when ways to assess construct are unfamiliar to people; and on item bias, i.e. when translation of items is either poor or ambiguous or may not be culturally acceptable (16).

Adaptation is primarily achieved by 2 processes: statistical, which employs quantitative procedures, and operative, which is a qualitative judgemental process that focuses on how and what kinds of adaptations have been done to ensure methodological equivalence with the original version of the instrument (17). The definitions which Flaherty et al. suggested (18) are outlined in Table1. Both qualitative and quantitative processes of adaptation are important and have their distinct methods of validation and ensuring scientific soundness. However, qualitative process takes precedence in adaptation of an instrument to a new culture or setting.

The adaptation of psychometric instruments to developing country settings is particularly challenging due to the lack of suitable experts in the subject $(19,20)$ and the general reluctance of the community to participate in research. There has been little research done to highlight such challenges and to suggest an adaptation procedure for developing countries, especially concerning instruments related to child behaviour. This paper explains a systematic process of qualitative adaptation of 3 parallel, multi-informant child behaviour problems instruments in a developing country, Pakistan, aiming to highlight the challenges faced during this process and to propose a logical model to attain a qualitative adaptation of psychometric instruments.

\section{Methods}

\section{Measures}

The tools selected for qualitative adaptation were the Achenbach System of Empirically Based Assessment (ASEBA) school-age instruments (21), i.e. the Child Behavior Checklist (CBCL) for ages $6-18$ years and its related parallel instruments; the Teacher's Report Form (TRF) for ages 6-18 years; and the Youth Self-Report (YSR) for ages 11-18 years. These instruments were originally developed in the United States of America (USA) and are widely used to measure child behaviour problems and are often considered as the gold standard for research and clinical work (22). They have been translated into more than 85 languages for use in developed and developing countries and have been used in more than 7000 international studies (23). They provide an assessment of a child's problems from different informants in different settings.

The YSR is a self-reported instrument for children to report their emotional and behaviour problems (105 problem items and 14 social desirability items) and competencies (20 items). The CBCL is for a caregiver to provide information about the child's competence (20 items) and problem behaviour (120 items). The TRF is a teacher-reported instrument with items related to academic and adaptive functioning in addition to 120 problem items. The competence items in CBCL and YSR provide information about a child's activities, social interactions and school performance, while problem items reflect the child's behaviour and emotional problems and are measured on a 3-point rating scale.

\section{Study setting and participants}

The adaptation process was carried out in Karachi, an urban cosmopolitan city of Pakistan, with a population representing all parts of the country. The study data were collected through

\begin{tabular}{ll}
\hline Table 1 Definitions of various types of methodological equivalences for adaptation of an instrument \\
\hline Type of equivalence & Definition \\
Conceptual equivalence & Measuring construct cross-culturally \\
Content equivalence & Ensuring item relevance in another culture \\
Semantic equivalence & Maintaining the meaning of items as given in original instrument \\
Technical equivalence & Ensuring reduction in method bias in the adapted instrument \\
Criterion equivalence & Assessing the ability of the instrument to discriminate between cases and non-cases (i.e. a \\
& quantitative assessment achieved via statistical processes) \\
\hline
\end{tabular}

Source: Flaherty JA, Gaviria FM, Pathak D, Mitchell T, Wintrob R, Richman JA, et al. (18). 
focus group discussions (FGDs) and final testing out. The study was done in collaboration with the local Teachers' Resource Centre. This is a teachers' professional capacity-building organization which has a huge network of member schools in Karachi, primarily private schools serving pupils of all socioeconomic levels, and was willing to be part of future studies or interventions based on the adapted instruments.

The Centre invited all co-education member schools that had both primary and secondary education curricula to participate in the study. Co-education was operationally defined as those schools in which male and female children study in the same classroom. The reason for including only coeducation schools was that the future empirical studies based on the adapted instruments could be compared crossculturally where schools provide equal opportunities to male and female children to study. Five schools showed an interest in participating in the study, but considering the qualitative nature of the study 2 schools were randomly selected for the FGDs, i.e. 1 school for the each of the 2 rounds of FGDs, and the rest of the schools were selected for the 2nd round of testing.

Participants (i.e. the informants of the study) were purposefully selected and comprised schoolchildren aged 11-18 years studying in grades 6 to 10 and mothers and female teachers of children aged 6-18 years. The decision to involve mothers participating in school-related activities rather than fathers was based on discussion with the Teachers' Resource Centre representatives and heads of member schools. The reason for inclusion of female teachers in the study was that most of the teachers in the education system of Pakistan, especially in school settings, are females.

\section{Study context}

The adaptation of the CBCL and related instruments was done primarily for a large-scale epidemiological study on behaviour problems among children in Karachi, Pakistan between the years 2009 to 2010 . This study was approved by the ethics review committee of Bridge Consultants Foundation, Pakistan, which is registered with the Office of Human Research Protection of the National Institutes of Health in the USA.

\section{Ethical considerations}

Informed consent to collect data from children, teachers and parents was received from schools after explaining the study's aim, objectives, procedures and instruments. Children and teachers were also informed about the study at the time of the data collection and although they were free to decide about participation they were encouraged to participate in the study by the school personnel. Formal invitation letters including information about the study and consent forms were sent to parents via schools. Parents who gave their consent were included in the study.

\section{Procedure}

The process of qualitative adaptation of the CBCL and related instruments was achieved through 6 discrete, sequential and iterative phases (Figure 1).

\section{Phase I: Selection phase}

The focus in phase I was on the selection of an appropriate instrument and developing a working group of multidisciplinary experts to ensure its cultural appropriateness. The authors selected the ASEBA instruments from all the leading instruments on the basis of their comprehensiveness in catering for a range of behaviour problems, their soundness in methodology used for their development and the evidence of their use in wider contexts $(24,25)$. Contact with the instruments' developer revealed the existence of Urdu translated versions needing systematic updates. Thus a collaborative, qualitative adaptation plan was mapped out. To form a working group, key national organizations were contacted to nominate 1 senior member from their respective departments. The final group comprised 6 multidisciplinary experts from the disciplines of child psychology, public health, epidemiology, education and bilingual linguistics in Urdu and English

\section{Phases II, III \& IV: Deliberation, al- teration and feasibility-iterative pro- cesses}

In the following phases the working group started deliberating on the suitability of the instruments in the Pakistani culture, determining the need for modifications in the provisional Urdu translations and moving on to alterations by suggesting suitable modifications. The working group also endorsed the selection of the ASEBA instruments and re-affirmed that those instruments could be compared cross-culturally. Finally, the feasibility of the modified instruments was checked with informants and the whole cycle was revised to eliminate non-feasible items.

The adaptation process also included a translation team and a field research team. The multidisciplinary translators were primarily involved in the alteration phase for forward and backward translation of the instruments but were also part of the final deliberation and iteration with the working group. The field research team was involved in checking the feasibility of the instruments in FGDs with informants and conducting a final test. Many rounds of meetings and FGDs followed as described below.

1st meeting with working group: To ascertain the cultural suitability of the instruments, the original version of each instrument was shared with experts and a consensus was reached about the necessary adaptation. All working group members were given a copy of the original English version and provisional translation of all 3 instruments (YSR, CBCL and TRF) and a working grid to specify agreement/disagreement about correctness of translations and sugges- 

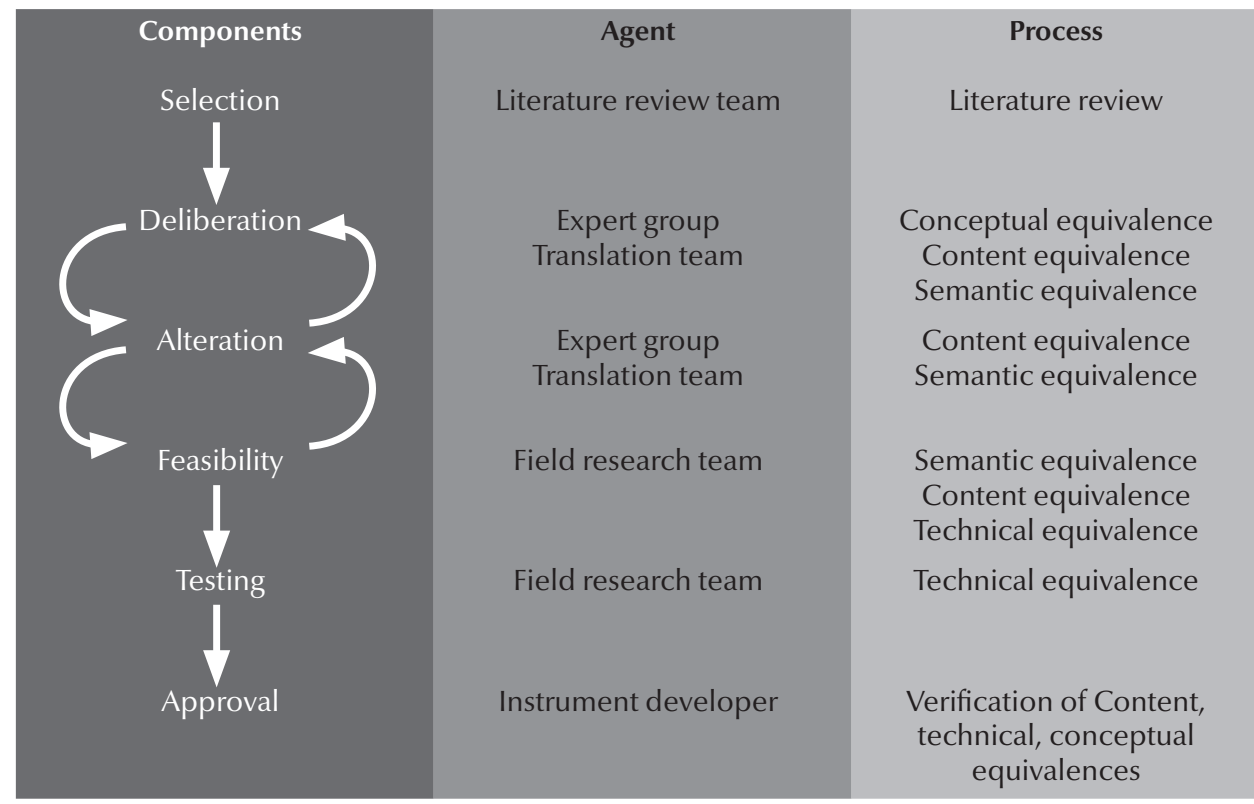

Reason
Valid instrument identification
$\begin{gathered}\text { Construct bias } \\ \text { Item bias }\end{gathered}$
$\begin{gathered}\text { Construct bias } \\ \text { Item bias }\end{gathered}$
Method bias
Method bias
Official version

Figure 1 Qualitative adaptation model of the psychometric instruments

tions for modifications, together with the reasons. Discussions were focused on determining cultural appropriateness and the equivalence of instrument items, including instructions for their administration, using dictionaries and thesauruses where necessary. All meetings were facilitated by the investigator and recorded by an observer.

1st round of FGDs with informants: FGDs were organized in 1 of the 2 schools randomly selected for FGDs and were conducted by a trained facilitator who was a clinical psychologist and had extensive experience and expertise in conducting and analysing FGDs with children and adults. The facilitator conducted 3 separate FGDs with male and female children aged 11-18 years, mothers of children aged 6-18 years and female teachers of children aged 6-18 years. The age range of mothers was 25-45 years and of teachers was 25-35 years. Each session included 7-8 participants who were purposefully selected. Through cognitive interviews all items and instructions of the provisionally translated instruments were discussed. Comprehension and cultural relevance were checked through explanation of items in their own words. We discussed all items one by one. For comprehension, participants were asked to describe and explain the item, i.e. they were asked to say that what they thought that item was about/saying or to repeat it in their own words that would reflect their interpretation of the item. They were also asked if there was any word in the item which they were not able to understand. For cultural relevance, they were asked if they were satisfied with the existing expression or would like to suggest any other easy and commonly used word/expression. Also they were asked if words/items were acceptable or offensive to them.

2nd meeting with working group: $\mathrm{Ob}$ servations and suggestions from the 1st round of FGDs were shared with the working group and this led to a consensus firstly to modify the instruments to make them equivalent to the original English versions and secondly to recheck these with informants.

2nd round of FGDs with informants: The 2 nd round of FGDs were carried out in another school and a new group of informants was purposefully selected, i.e. male and female children aged $11-$
18 years and mothers and female teachers of children aged $6-18$ years. The age range of mothers was $25-40$ years while teachers were aged $30-40$ years. Provisional translations including suggested modifications were discussed. Suggestions for cultural adaptation of the examples in the instruments were also noted. The facilitator who conducted the first round of FGDs facilitated the second round as well.

Based on the above procedure 3 detailed documents for the YSR, CBCL and TRF respectively were produced indicating each item number, provisional translation, suggested modifications in translation and reason for modification. Documents with details of procedures were shared with the developer and a formal licence was received for updating the translations.

\section{Forward translation of instruments:} Three bilingual English-Urdu translators, with backgrounds in psychology, public health and linguistics respectively, translated the instruments as a group, keeping in mind all suggestions from the working group and FGDs. They ensured conceptual equivalence (translating the same concept), semantic 
equivalence (identifying appropriate words), content equivalence (ensuring item relevance) and technical equivalence (ensuring syntax, grammar, layout and format of the original English version).

Back translation: All the translated instruments were then back translated to English by an independent bilingual linguist who was blind to the original English instruments.

Final review by the working group: All the original, translated and back translated instruments were then shared with the working group for their feedback and agreement to initiate testing.

\section{Phases V and VI: Testing and ap- proval}

Testing and finalization of instruments: The adapted instruments were finally tested on 100 male and female children aged $6-18$ years. Of them, for 70 male and female children aged 11-18 years the informants were the children themselves, their mothers and their 6 teachers, whereas for 30 male and female children aged 6-10 years, their mothers and 3 teachers were the informants. The age range of mothers was $25-45$ years and of teachers was 25-40 years.

Testing was carried out in the randomly selected 3 schools of the Teachers' Resource Centre. The participants were instructed by the field research team to complete the instruments. Immediately after the participants handed over the complete instruments to the team they were asked questions regarding the comprehension of instructions, meaning of items, ease of language, cultural relevance and ease with format and layout. The field research team that conducted the testing comprised 6 members with master's degrees in psychology and education with prior research experience of working with children and adults in communities. The field team was provided with training in interview and communication skills before data collection. Out of 6 members of the team 3 conducted the testing with children, 2 with mothers and 1 with teachers.

\section{Approval}

During testing, the field research team identified a few ambiguities in the adapted instruments, which were then corrected and incorporated by the investigators into the instruments after the testing. Final translations and back translations of adapted instruments were then shared with the instruments' developer in order to obtain approval as an official version of ASEBA.

\section{Results}

\section{Phase 1: Selection}

Child behaviour problems have been assessed through various approaches, but rating scales are the most commonly used assessment approach in epidemiological research and interventions (26) because of their standardized format for assessing, scoring and interpreting behaviour (25). The rating scales include generalpurpose instruments assessing all type of behaviour problems in the general population (25) and those measuring specific problems such as depression (27). Although there are other general-purpose instruments to measure child behaviour problems (28-30), the ASEBA tools were chosen by our panel because of their comprehensiveness in assessing child behaviour problems, the sound methodology of their development and established international reputation for epidemiological studies.

\section{Phase II-IV: Deliberation, alteration and feasibility}

\section{1st meeting with working group}

Besides checking the instruments for equivalence with the original English versions the working group also identified numerous typing errors and use of Persian and Arabic origin words in the provisional translations. Some examples of modifications suggested by the working group for equivalence were as follows:

- Conceptual equivalence: YSR problem item 15 "I am pretty honest" was incorrectly translated as main aksar sach bolta hon ("I often speak truth"). However, the appropriate and literal translation of "honest" could be imaandaar, because speaking truth is part of honesty (imaandari), as in other attributes such as trustworthiness and conscientious. In the same item "pretty" was also incorrectly translated as aksar ("often"); the correct translation of "pretty" is kaafi.

- Content equivalence: CBCL problem item 11 "clings to adults" was not translated in the provisional Urdu version. The translation could be $b a-$ roon se chipkta/chipakte hai. This addition was important to make the item more comprehensible.

- Semantic equivalence: CBCL problem item 8 "for long" was translated as taweel arse kay liye, in which taweel is an Arabic origin word and taweel arse generally refers to years. Ziyada dair tak gives the exact meaning of the English expression and is also commonly used.

- Technical equivalence: TRF item 24 "pupils" was translated as talaba which is the plural of talib e ilm ("male student"). In English language the plural is used for both male and female students, whereas in Urdu, talibaat, the plural of taliba, is used for female students. Therefore it was suggested that talaba or talibaat be used instead of mentioning only talaba.

\section{1st round of FGDs with informants}

FGDs with children, mothers and teachers highlighted numerous items and instructions which were either difficult to understand or misunderstood. Some examples are given as follows:

- Semantic equivalence: YSR problem item 13 "confusion" was translated as tazabzub, which is a very difficult word 
for children to understand because it is an uncommon Arabic origin word. However, uljhan, a synonym of taza$b z u b$, is widely used and understood.

- Semantic equivalence: TRF problem item 86 "irritable" was translated as tunak mizaaj, which is a difficult word for teachers as it is an uncommon Persian-origin word, whereas chirchi$\mathrm{ra}$ is a synonym of tunuk mizaaj and is very easy to understand.

- Technical equivalence: unnecessary grammatical changes in items caused some offence to participants; for example, CBCL problem item 43 "lying and cheating" was translated as liar and cheater, wo jhota/jhoti aur dhokaybaz hai.

- Content equivalence: participants suggested add a few more culturally relevant examples such as sports like cricket, hockey and football in CBCL and YSR competence item 1 and also suggested deleting fishing as it is not a common local sport.

- Technical equivalence: all participants were confused about the instructions for the problem items. "True" was translated as sach ("truth") and they were confused that if they marked that a certain behaviour never happened, it would it be considered as lying.

Participants also suggested retaining items related to sex and alcohol in spite of the cultural taboos concerning these.

\section{2nd meeting with working group}

This meeting revealed and discussed issues arising in translation with respect to misunderstandings and use of potentially offensive and morally connotated words in items. The working group endorsed the informants' suggestions and agreed to modify offensive translations while maintaining the equivalence with the original items. Some examples are as follows:

- After suggestions from informants to add the name of local sports in YSR and CBCL competence item 1 it was decided to include them with the ones mentioned in the original English version and to recheck it in the 2 nd round of FGDs.

- Misinterpretation of instruction and response categories of the problem items in the CBCL, YSR and TRF was identified as due to the translation of "true" to sach, which means "truth", and "not true" as sach nahin, which means "lie". This seemed to have a moral connotation to respondents. However, the purpose of the items in the instruments was to determine whether a certain behaviour is relevant to the child and the extent of its applicability. Therefore translating "true" as sach would be inappropriate, while the suggested translation sahi seemed appropriate as it could enable the informant to see the applicability of the items by agreeing, disagreeing or somewhat agreeing without invoking the moral implications of being "truthful".

The working group also endorsed retaining items pertaining to sex and alcohol.

\section{2nd round of FGDs with informants}

In the 2 nd round of FGDs the new groups of informants endorsed the modified translations and the retention of culturally relevant examples.

\section{Forward translation of instruments}

During translation, the primary focus was on semantic equivalence while incorporating inputs from the working group and FGDs regarding other types of equivalence as well. It was ensured that layout, format, item placement, sequence and scoring patterns were equivalent. Items were translated in functional and simple language to suit the requirement of self-report instruments. It was ensured that items were used with supportive expressions that made them useable for both sexes, as required in Urdu language.

\section{Final review from the working group}

The working group reviewed and discussed the translations and back translations with the original English version with the translators and approved them for final testing.

\section{Phase V: Testing}

During testing, informants found the adapted instruments relevant and understandable but a few typing errors were identified that were corrected and incorporated in the instruments after the testing.

\section{Phase VI: Approval}

Final translation and back translation of instruments were then shared with the instruments' developer who checked back translations with the original English version of the instruments to ensure equivalence. He further confirmed the equivalence of adapted instruments and also highlighted an important point by suggesting replacing English as an academic subject with Urdu in CBCL and YSR item VII-1, assuming that English might not be taught in Pakistan. However, we finally included Urdu along with English with the rationale that in Pakistan we have Urdu and English language schools so children belonging to both type of education systems could easily relate to it.

\section{Discussion}

Qualitative adaptation is a systematic and rigorous process as compared with a simple translation of a psychometric instrument, especially in the context of a developing country. But, once achieved, the process ensures the quality of research findings as compared with more compromised adaptations $(31,32)$. Since there is no consensus on the best practice to be employed for qualitative adaptation (17), we attempted a systematic approach to satisfy multiple equivalence needs of an adapted version of the child behaviour problem instruments and proposed a model of qualitative adaptation of psychometric instruments for developing countries 
(Figure 1). The process of qualitative adaptation comprised 6 phases and employed many arduous iterations until the instruments were technically equivalent to the original English versions, with minimal potential biases. There follows a brief account of the key logistic and other challenges faced during the adaptation process.

Language is a complex social phenomenon whose content and structure is continually evolving through historical, cultural and socioeconomic influences. Vocabulary expansion in languages sometimes borrows words from multiple other linguistic sources; consequently a choice of words sometimes exist even for the same experience. The selection of the specific word depends on the sociocultural background of the user or listener in order to capture the specific nuance in the communication. This understanding is particularly important for the qualitative adaptation of a psychometric instrument. The Urdu language vocabulary contains a range of words borrowed from native languages such as Punjabi, Pashto and Sindhi and regional languages such as Arabic, Persian Sanskrit and Turkish. Its translational vocabulary and syntactical structure changes with the sophistication of language use. Various alternatives are available for the same concept and therefore choice of words requires careful deliberation. One of the key challenges of this project was to come up with a translation that achieved uniform comprehension across all social classes.

The other technical challenge was synchronization of multi-informant instruments that had common items. Multi-informant instruments obtain the views of different informants about the same child's behaviour. During adaptation, using the YSR as the reference, it was necessary to ensure that translations of common items and terminologies be consistent across all the instruments, as all informants would be reporting about the same child and therefore interpretation of such items should be consistent. After extensive discussions with informants, words were obtained that were commonly understandable and equivalent with the original English versions.

Consistent with studies conducted to adapt various instruments in developing countries (33,34), another challenge was to ensure all types of equivalences - semantic, conceptual, content and technical — with the original English version. For semantic equivalence, the fact that these instruments were to be completed via self-reporting meant that we needed to identify commonly used words so that informants would not need further clarification. Conceptual and content equivalences were ensured by critical discussion of all items with the working group and FGD informants. However, some changes were made by adding culturally relevant examples to facilitate understanding. After careful exploration in FGDs, testing and discussions with the working group, we decided to retain those items related to sex and alcohol. The logic for this was that it would not be a good idea to assume that these were not culturally relevant (due to low frequency or under-reporting) or exclude any problem item, especially when such problems do exist even in a conservative society like Pakistan. To ensure technical equivalence, we checked if informants were comfortable with the format of item presentation, response categories and instructions.

Developing a working group of experts (35) who were working from a public health perspective with relevant experience and expertise was an uphill task. As there is a general scarcity of mental health experts in developing countries $(36,37)$ very few people are working exclusively in the area of child behaviour and even fewer had practical research experience. Moreover, a majority of the experts work normally in isolation in their respective fields. However, formation of multidisciplinary groups with experience in psychology- and public health-related initiatives played a key role in devising a better adapted instrument.

The qualitative adaptation phases containing cycles of deliberation, alteration and checking of feasibility played a crucial role in achieving the instruments' equivalence and minimizing biases. These phases were the result of effort and collaboration by 3 teams. The working group shared their candid views by identifying errors in provisional translations and suggesting appropriate modifications, with reasons. FGDs (38), conducted by the field research team using cognitive interviews (39) with informants helped not only in determining informants' understanding and cultural relevance of items but also in getting their inputs to create appropriate instructions and culturally relevant items. Finally, the team of multidisciplinary translators, which was considered better than independent translators (32), discussed all items and instructions and reached a consensus, while acknowledging the inputs from the working group and FGDs and ensuring congruent cross-cultural translation (18,40).

An important addition to this adaptation was the involvement of the developer of the instruments (35). It was an important practice to apply the technical, conceptual and even content understanding of the original developer of the instrument in the translated version. Finally, further authentication of the quality of adaptation was achieved via the formal approval of the developer himself.

FGDs and the final testing that were conducted in a school setting ensured that the adapted instruments had relevance for local informants. It is pertinent to mention that schools in Pakistan are generally not involved in research or child assessments. However, our collaboration with the teachers' professional capacity-building organization (the Teachers' Resource Centre) was extremely useful and played a vital role in convincing the schools' administration 
and encouraging parents, children and teachers to participate actively in the research.

It is important to highlight that the child behaviour problems instruments have been translated in both developed and developing countries but, interestingly, among developing countries only a few have translated all 3 parallel school age instruments available, i.e. the YSR $11-18$, CBCL 6-18 and TRF 6-18, to achieve multi-informant views of a child's behaviour (23). Moreover, very limited validation studies from developing countries are available that have reported the qualitative adaptation of all 3 parallel school-age instruments (41). Our study not only illustrates the rigorous and iterative process of adaptation of all 3 parallel instruments conducted in collaboration with the instruments' developer but also take a step forward in proposing a systematic model to conduct qualitative adaptation in a developing-country setting.

It is important to mention here that investigators were involved in every phase of adaptation to monitor quality and ensure smooth transition of this process.
A limitation of the study was that the instruments were tested on informants only in Karachi, a cosmopolitan city that has people from all ethnic and socioeconomic backgrounds. The relevance of the adapted version is for the communities which have similar linguistic and cultural characteristics.

Our primary focus was to conduct and highlight the steps of the qualitative adaptation of child behaviour problems instruments from a developing country context. Certainly, quantitative adaptation is required that will further ensure the validity of the instrument. However, we believe that it is necessary to determine cultural relevance and to conduct a thorough qualitative adaptation of the instruments before moving on to determining their psychometrics (17).

\section{Conclusions}

To ensure theinstruments' quality and equivalence for cross-cultural research in developing countries, we propose a rigorous and systematic model to achieve effective cultural adaptation of psychometric instruments. This process followed 6 meticulous phases in order to achieve conceptual, content, semantic and technical equivalences with the original English version. We conceived and refined this model by conducting qualitative adaptation of 3 parallel, multi-informant child behaviour problems instruments in Pakistan.

\section{Acknowledgements}

We are very grateful to the working group of experts, the translation team, field team, Teachers' Resource Centre and schools, especially the children, teachers and parents, for participating in the adaptation process. We are also thankful to Dr Thomas Achenbach for his keen involvement and technical advice during this process of adaptation. We appreciate the support of Ramani Sunderaju from ASEBA.We are also grateful to Dr Rakshanda Hussain for giving her valuable feedback on the manuscript.

Funding: Not applicable.

Competing interests: None declared.

\section{References}

1. O'Connell ME, Boat T, Warner KE, editors. Preventing mental, emotional, and behavioral disorders among young people: progress and possibilities. Washington: National Academies Press; 2009.

2. Prevention of mental disorders: effective interventions and policy options. Summary report. A report of the World Health Organization, Department of Mental Health and Substance Abuse in collaboration with the Prevention Research Centre of the Universities of Nijmegen and Maastricht. Geneva: World Health Organization; 2004 (http://www.who.int/ mental_health/evidence/en/prevention_of_mental_disorders_sr.pdf, accessed 5 May 2014).

3. Eapen V, Jairam R. Integration of child mental health services to primary care: challenges and opportunities. Ment Health Fam Med. 2009 Mar;6(1):43-8.

4. Knapp M, King D, Healey A, Thomas C. Economic outcomes in adulthood and their associations with antisocial conduct, attention deficit and anxiety problems in childhood. J Ment Health Policy Econ. 2011 Sep;14(3):137-47. PMID:22116171

5. Tremblay RE. Developmental origins of disruptive behaviour problems: the 'original $\sin$ ' hypothesis, epigenetics and their consequences for prevention. J Child Psychol Psychiatry. 2010 Apr;51(4):341-67. PMID:20146751
6. Bongers IL, Koot HM, van der Ende J, Verhulst FC. Predicting young adult social functioning from developmental trajectories of externalizing behaviour. Psychol Med. 2008 Jul;38(7):989-99. PMID:18047767

7. Fergusson DM, Horwood LJ, Ridder EM. Show me the child at seven: the consequences of conduct problems in childhood for psychosocial functioning in adulthood. J Child Psychol Psychiatry. 2005 Aug;46(8):837-49. PMID:16033632

8. Koot HM, Verhulst FC. Prevalence of problem behavior in Dutch children aged 2-3. Acta Psychiatr Scand Suppl. 1991;367:1-37. PMID:1897379

9. Shenoy J, Kapur M, Kaliaperumal VG. Psychological disturbance among 5- to 8-year-old school children: a study from India. Soc Psychiatry Psychiatr Epidemiol. 1998 Feb;33(2):66-73. PMID:9503989

10. Javo C, Rønning JA, Handegård BH, Rudmin FW. Social competence and emotional/behavioral problems in a birth cohort of Sami and Norwegian preadolescents in Arctic Norway as reported by mothers and teachers. Nord J Psychiatry. 2009;63(2):178-87. PMID:19214866

11. Woo BS, Ng TP, Fung DS, Chan YH, Lee YP, Koh JB, et al. Emotional and behavioural problems in Singaporean children 
based on parent, teacher and child reports. Singapore Med J. 2007 Dec;48(12):1100-6. PMID:18043836

12. Roberts RE, Attkisson CC, Rosenblatt A. Prevalence of psychopathology among children and adolescents. Am J Psychiatry. 1998 Jun;155(6):715-25. PMID:9619142

13. Avan B, Richter LM, Ramchandani PG, Norris SA, Stein A. Maternal postnatal depression and children's growth and behaviour during the early years of life: exploring the interaction between physical and mental health. Arch Dis Child. 2010 Sep;95(9):690-5. PMID:20660522

14. Kieling $\mathrm{C}$, Baker-Henningham $\mathrm{H}$, Belfer M, Conti $\mathrm{G}$, Ertem I, Omigbodun O, et al. Child and adolescent mental health worldwide: evidence for action. Lancet. 2011 Oct 22;378(9801):1515-25. PMID:22008427

15. Bhui K, Mohamud S, Warfa N, Craig TJ, Stansfeld SA. Cultural adaptation of mental health measures: improving the quality of clinical practice and research. Br J Psychiatry. 2003 Sep;183:184-6. PMID:12948986

16. Vijver FJRvd. Poortinga YH. Conceptual and methodological Issues in adapting tests. In: Hambleton RK, Merenda PF, Spielberger $\mathrm{CD}$, editors. Adapting educational and psychological tests for cross cultural assessment. New Jersey: Lawrence Erlbaum Associates; 2005.

17. Malda M. Vijver FJRvd, Srinivasan K, Transler C, Sukumar P, Rao K. Adapting a cognitive test for a different culture: An illustration of qualitative procedures. Psychology Science Quarterly. 2008;50(4):451-68.

18. Flaherty JA, Gaviria FM, Pathak D, Mitchell T, Wintrob R, Richman JA, et al. Developing instruments for cross-cultural psychiatric research. J Nerv Ment Dis. 1988 May;176(5):257-63. PMID:3367140

19. 19. Bruckner TA, Scheffler RM, Shen G, Yoon J, Chisholm D, Morris J, et al. The mental health workforce gap in low- and middle-income countries: a needs-based approach. Bull World Health Organ. 2011 Mar 1;89(3):184-94. PMID:21379414

20. Saraceno B, van Ommeren M, Batniji R, Cohen A, Gureje O, Mahoney J, et al. Barriers to improvement of mental health services in low-income and middle-income countries. Lancet. 2007 Sep 29;370(9593):1164-74. PMID:17804061

21. Achenbach TM, Rescorla LA. Manual for the ASEBA school-age forms and profiles. Burlington (VT): University of Vermont, Research Center for Children, Youth, and Families; 2001.

22. Dulcan MK, editor. Dulcan's textbook of child and adolescent psychiatry. Arlington (VA): American Psychiatric Publishing; 2010.

23. The ASEBA approach [Internet]. Burlington (VT): Achenbach System of Empirically Based Assessment (http://www.aseba. org/, accessed 5 May 2014).

24. ASEBA research updates from around the world [Internet]. Burlington (VT): Achenbach System of Empirically Based Assessment (http://www.aseba.org/research/research.html, accessed 5 May 2014).

25. Merrell KW. Behavioral, social and emotional assessment of children and adolescents. 3rd ed. New York: Lawrence Erlbaum Associates; 2008.

26. Gelfand DM, Drew CJ. Understanding child behavior disorders. 4th ed. Belmont, CA: Wadsworth Publishing; 2003.
27. Reynolds WM. Reynolds child depression scale. Odessa (FL): Psychological Assessment Resources; 1989.

28. Reynolds CR, Kamphaus RW. Behavior assessment system for children. 2nd ed. Circle Pines (MN): AGS Publishing; 2004.

29. Bracken BA, Keith LK. Clinical assessment of behavior. Lutz (FL): Psychological Assessment Resources; 2004.

30. Gadow KD, Sprafkin J. Childhood symptom inventory-4: screening and norms manual. Stony Brook (NY): Checkmate Plus; 2002.

31. Merenda PF. Cross-cultural adaptation and educational and psychological testing. In: Hambleton RK, Merenda PF, Spielberger $\mathrm{CD}$, editors. Adapting educational and psychological tests for cross-cultural assessment. Mahwah (NJ): Lawrence Erlbaum Associates; 2005.

32. Harkness J, Pennell B-E. Schoua-Glusberg Au. Survey questionnaire translation and assessment. In: Presser S, Rothgeb JM, Couper MP, Lessler JT, Martin E, Martin J, et al., editors. Methods for testing and evaluating survey questionnaires. Hoboken (NJ): John Wiley \& Sons; 2004.

33. Matías-Carrelo LE, Chávez LM, Negrón G, Canino G, AguilarGaxiola S, Hoppe S. The Spanish translation and cultural adaptation of five mental health outcome measures. Cult Med Psychiatry. 2003 Sep;27(3):291-313. PMID:14510096

34. Smit J, van den Berg CE, Bekker LG, Seedat S, Stein DJ. Translation and cross-cultural adaptation of a mental health battery in an African setting. Afr Health Sci. 2006 Dec;6(4):215-22. PMID:17604510

35. Beaton DE, Bombardier C, Guillemin F, Ferraz MB. Guidelines for the process of cross-cultural adaptation of self-report measures. Spine (Phila Pa 1976). 2000 Dec 15;25(24):3186-91. PMID:11124735

36. Patel V, Flisher AJ, Hetrick S, McGorry P. Mental health of young people: a global public-health challenge. Lancet. 2007 Apr 14;369(9569):1302-13. PMID:17434406

37. Okasha A, Karam E, Okasha T. Mental health services in the Arab world. World Psychiatry. 2012 Feb;11(1):52-4. PMID:22295010

38. Knudsen HC, Vázquez-Barquero JL, Welcher B, Gaite L, Becker $\mathrm{T}$, Chisholm D, et al. Translation and cross-cultural adaptation of outcome measurements for schizophrenia. EPSILON Study 2. European Psychiatric Services: inputs linked to outcome domains and needs. Br J Psychiatry Suppl. 2000; (39):s8-14. PMID:10945072

39. Process of translation and adaptation of instruments. Management of substance abuse. Geneva: World Health Organization; 2012 (http://www.who.int/substance_abuse/ research_tools/translation/en/, accessed 5 May 2014).

40. Sartorius N, Kuyken W. Translation of health status instruments. In: Orley KW, editor. Quality of life assessment in health care settings. Berlin: Springer; 1994.

41. Bordin IA, Rocha MM, Paula CS, Teixeira MC, Achenbach TM, Rescorla LA, et al. Child Behavior Checklist (CBCL), Youth SelfReport (YSR) and Teacher's Report Form (TRF): an overview of the development of the original and Brazilian versions. Cad Saude Publica. 2013 Jan;29(1):13-28. PMID:23370021 